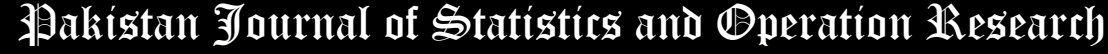

\section{The Poisson Topp Leone Generator of Distributions for Lifetime Data: Theory, Characterizations and Applications}

\author{
Faton Merovci ${ }^{1}$, Haitham M. Yousof ${ }^{2}$ and G.G. Hamedani ${ }^{3}$
}

1. Faculty of Mechanical and Computer Engineering, University of Mitrovica "Isa Boletini”, Kosovo, faton.merovci@umib.net

2. Department of Statistics, Mathematics and Insurance, Benha University, Egypt, haitham.yousof@fcom.bu.edu.eg

3. Department of Mathematics, Statistics and Computer Science, Marquette University, USA, gholamhoss.hamedani@marquette.edu

\begin{abstract}
:
We study a new family of distributions defined by the minimum of the Poisson random number of independent identically distributed random variables having a Topp Leone-G distribution (see Rezaei et al., (2016)). Some mathematical properties of the new family including ordinary and incomplete moments, quantile and generating functions, mean deviations, order statistics, reliability and entropies are derived. Maximum likelihood estimation of the model parameters is investigated. Some special models of the new family are discussed. An application is carried out on real data set applications sets to show the potentiality of the proposed family.
\end{abstract}

Keywords: Topp Leone-G Family, Order Statistics, Maximum Likelihood Estimation, Quantile function, Generating Function, Moments.

Mathematical Subject Classification: 60E05, 97K50, 62N02.

\section{Introduction}

The statistical literature contains many new classes of distributions which have been constructed by extending common families of continuous distributions and give more flexibility by adding one or more parameters to the baseline model. These new families have been used for modeling data in many applied areas such as engineering, economics, biological studies and environmental sciences. So, several classes of distributions have been constructed by extending common families of continuous distributions. These generalized distributions give more flexibility by adding one "or more" parameters to the baseline model. They were pioneered by Gupta et al. (1998) who proposed the exponentiated-G class, which consists of raising the cumulative distribution function (CDF) to a positive power parameter. Many other classes can be cited such as the Marshall-Olkin-G family by Marshall and Olkin (1997), beta generalized-G family by Eugene et al. (2002), transmuted exponentiated generalized-G family by Yousof et al. (2015), generalized transmuted-G by Nofal et al. (2017), the Topp Leone generated family by Rezaei et al. (2017), exponentiated transmuted-G family by Merovci et al. (2017), Topp-Leone Odd Log-Logistic Family of Distributions by Brito et al. (2017), Burr X-G by Yousof et al. (2017), exponentiated generalized-G Poisson family by Aryal and Yousof (2017), type I general exponential class of distributions by Hamedani et al. (2017), Burr XII system of densities by Cordeiro et al. (2018), exponential Lindley odd log-logistic G family by Korkmaz et al. (2018a), Marshall-Olkin generalized G Poisson family by Korkmaz et al. (2018b), Burr-Hatke family of distributions by Yousof et al. (2018), a new extended $\mathrm{G}$ family by Hamedani et al. (2018), Type II general exponential class of distributions by Hamedani et al. (2019) and odd Nadarajah-Haghighi family of distributions by Nascimento et al. (2019), the Weibull G Poisson family by Yousof et al.(2020), among other. 
Due to Rezaei et al. (2017), the cumulative distribution function (CDF) and the probability density function (PDF) of the Topp Leone (TL) generated family of distributions distribution specified by

and

$$
H(x ; \alpha, \psi)=\{G(x ; \psi)[2-G(x ; \psi)]\}^{\alpha}=G(x ; \psi)^{\alpha}[2-G(x ; \psi)]^{\alpha},
$$

$$
h(x ; \alpha, \psi)=2 \alpha g(x ; \psi) G(x ; \psi)^{\alpha-1}[1-G(x ; \psi)][2-G(x ; \psi)]^{\alpha-1},
$$

respectively. Suppose $Z_{1}, \ldots, Z_{N}$ be independent identically random variable (iid) with common CDF Topp Leone-G and $N$ be random variable with

$$
P(N=n)=\frac{1}{e^{\lambda}-1} \times \frac{\lambda^{n}}{n !}, n=1,2, \ldots, \lambda>0,
$$

and define $M_{N}=\max \left\{Z_{1}, \ldots, Z_{N}\right\}$ then

$$
F(x)=\sum_{n=0}^{\infty} p\left(M_{N} \leq x \mid N=n\right) p(N=n) .
$$

Equation (3) can be expressed as

$$
F(x)=\sum_{n=0}^{\infty}\left\{\frac{1-\exp [-\lambda H(x ; \alpha, \psi)]}{e^{\lambda}-1}\right\}^{n} \frac{1}{e^{\lambda}-1} \frac{\lambda^{n}}{n !}
$$

Using equations (2) and the last equation, we can write

$$
F(x)=\frac{1-\exp \left\{-\lambda G(x ; \psi)^{\alpha}[2-G(x ; \psi)]^{\alpha}\right\}}{\left(1-e^{-\lambda}\right)} .
$$

Equation (4) is called Poisson Topp Leone-G (PTL-G) family of distributions. Several new models can be generated by considering special distributions for $G(x)$. The corresponding PDF of reduces to

$$
f(x)=\frac{2 \lambda \alpha g(x ; \psi) G(x ; \psi)^{\alpha-1}[1-G(x ; \psi)][2-G(x ; \psi)]^{\alpha-1}}{\left(1-e^{-\lambda}\right) \exp \left\{\lambda G(x ; \psi)^{\alpha}[2-G(x ; \psi)]^{\alpha}\right\}} .
$$

Henceforward $G(x ; \psi)=G(x)$ and $g(x ; \psi)=g(x)$. Now we can provide a useful linear representation for the PTL-G density function in. Expanding the quantity $\exp \left\{-\lambda G(x)^{\alpha}[2-G(x)]^{\alpha}\right\}$ in power series, we can write

$$
f(x)=\sum_{i=0}^{\infty} \frac{\lambda^{i+1} \alpha 2^{\alpha(i+1)}(-1)^{i} g(x)[1-G(x)]}{i !\left(1-e^{-\lambda}\right) G(x)^{-[\alpha(i+1)-1]}}\left[1-\frac{G(x)}{2}\right]^{\alpha(i+1)-1} .
$$

Consider the power series

$$
(1-n)^{c}=\sum_{j=0}^{\infty} \frac{(-1)^{j} \Gamma(1+c)}{j ! \Gamma(1+c-j)} n^{j}
$$

which holds for $|z|<1$ and $b>0$ real non-integer. Using the power series in and after some algebra the PDF of the PTL-G class in can be expressed as

$$
f(x)=\sum_{i, j=0}^{\infty}\left\{\Upsilon_{i, j} \pi_{\alpha(i+1)+j}(x)-\Upsilon_{i, j}^{*} \pi_{\alpha(i+1)+j+1}(x)\right\},
$$

where $\pi_{\gamma}(x)=\gamma g(x) G(x)^{\gamma-1}$.

$$
\begin{aligned}
& Y_{i, j}=\frac{\alpha \lambda^{i+1}(-1)^{i+j} 2^{\alpha(i+1)-j}}{i !\left(1-e^{-\lambda}\right)[\alpha(i+1)+j]}\left(\begin{array}{c}
\alpha(i+1)-1 \\
j
\end{array}\right) \text { and } \\
& Y_{i, j}^{*}=\frac{\alpha \lambda^{i+1}(-1)^{i+j} 2^{\alpha(i+1)-j}}{i !\left(1-e^{-\lambda}\right)[\alpha(i+1)+j+1]}\left(\begin{array}{c}
\alpha(i+1)-1 \\
j
\end{array}\right) .
\end{aligned}
$$

Equation (8) reveals that the density of $X$ can be expressed as a linear representation of exp-G densities. So, several mathematical properties of the new family can be obtained by knowing those of the exp-G distribution. The CDF of the PTL-G family can also be expressed as a mixture of exp-G densities. By integrating, we obtain the same mixture representation 


$$
f(x)=\sum_{i, j=0}^{\infty}\left\{Y_{i, j} \Pi_{\alpha(i+1)+j}(x)-Y_{i, j}^{*} \Pi_{\alpha(i+1)+j+1}(x)\right\},
$$

where $\Pi_{\gamma}(x)$ is the CDF of the exp-G family with power parameter $(\gamma)$. The rest of the paper is outlined as follows. In Section 2, we define three special models and provide the plots of their PDF's and hazard rate functions (HRF's). In Section 3, we derive some of its mathematical properties including probability weighted moments (PWMs), residual life and reversed residual life functions, ordinary, incomplete moments and generating functions, finally order statistics and their moments are introduced at the end of the section. Some characterizations results are provided in Section 4. Maximum likelihood estimation of the model parameters is addressed in Section 5. In section 6, simulation results to assess the performance of the proposed maximum likelihood estimation procedure are discussed. In Section 7 , we provide the applications to real data to illustrate the importance of the new family. Finally, some concluding remarks are presented in Section 8.

\section{Special models}

\subsection{PTL-Exponential (PTL-E) distribution}

The parent exponential distribution has PDF and CDF given, respectively, by

The CDF of PTE-Exponential distribution if given by $(x>0)$

$$
G(x, b)=1-\exp (-b x) \text {. }
$$

$$
F(x)=\frac{1-\exp \left\{-\lambda(1-\exp (-b x))^{\alpha}[1+\exp (-b x)]^{\alpha}\right\}}{\left(1-e^{-\lambda}\right)} .
$$

Figures 1 and 2 illustrate some of the possible shapes of the PDF and HRF of the PTL-E distribution.

\subsection{The PTL-Weibull (PTL-W) distribution}

The parent Weibull distribution (see Weibull (1951)) has CDF given by

The CDF of PTL-W distribution is given by $(x>0)$

$$
G(x ; a, b)=1-\exp \left(-b x^{a}\right) .
$$

$$
F(x)=\frac{1-\exp \left\{-\lambda\left(1-\exp \left(-b x^{a}\right)\right)^{\alpha}\left[1+\exp \left(-b x^{a}\right)\right]^{\alpha}\right\}}{\left(1-e^{-\lambda}\right)} .
$$

Figures 3 and 4 illustrates possible shapes of the PDF and HRF for some PTL-W distributions.

\subsection{The PTL-generalized half normal (PTL-GHN) distribution}

The parent generalized half normal distribution has CDF given by

The CDF and PDF of PTL-GHN distribution are given by

$$
G(x, a, b)=2 \Phi\left[\left(\frac{x}{a}\right)^{b}\right]-1 .
$$

$$
F(x)=\frac{1-\exp \left\{-\lambda G\left\{2 \Phi\left[\left(\frac{x}{a}\right)^{b}\right]-1\right\}^{\alpha}\left(2-\left\{2 \Phi\left[\left(\frac{x}{a}\right)^{b}\right]-1\right\}\right)^{\alpha}\right\}}{\left(1-e^{-\lambda}\right)}
$$

\section{Mathematical properties}

\subsection{Probability weighted moments}

The PWMs are expectations of certain functions of a random variable and they can be defined for any random variable whose ordinary moments exist. The PWM method can generally be used for estimating parameters of a distribution whose inverse form cannot be expressed explicitly. The $(s, r)$ th PWM of $X$ following the PTL-G family, say $\rho_{s, r}$, is formally defined by

$$
\rho_{s, r}=E\left\{X^{s} F(X)^{r}\right\}=\int_{-\infty}^{\infty} x^{s} F(x)^{r} f(x) d x .
$$

Using equations (5) and (6), we can write

$$
f(x) F(x)^{r}=\sum_{i, j=0}^{\infty}\left\{p_{i, j} \pi_{\alpha(i+1)+j}(x)-p_{i, j}^{*} \pi_{\alpha(i+1)+j+1}(x)\right\},
$$


where

and

$$
p_{i, j}=\sum_{k=0}^{\infty} \frac{2^{\alpha(i+1)-j} \alpha \lambda^{i+1}(-1)^{i+j+k}(k+1)^{i}\left(\begin{array}{l}
r \\
k
\end{array}\right)\left(\begin{array}{c}
\alpha(i+1)-1 \\
j
\end{array}\right)}{i !\left(1-e^{-\lambda}\right)^{r+1}[\alpha(i+1)+j]}
$$

Then, the $(s, r)$ th PWM of $X$ can be expressed as

$$
p_{i, j}^{*}=\sum_{k=0}^{\infty} \frac{2^{\alpha(i+1)-j} \alpha \lambda^{i+1}(-1)^{i+j+k}(k+1)^{i}\left(\begin{array}{l}
r \\
k
\end{array}\right)\left(\begin{array}{c}
\alpha(i+1)-1 \\
j
\end{array}\right)}{i !\left(1-e^{-\lambda}\right)^{r+1}[\alpha(i+1)+j+1]} .
$$

$$
\rho_{s, r}=\sum_{i, j=0}^{\infty}\left\{p_{i, j} E\left(Y_{\alpha(i+1)+j}^{s}\right)-p_{i, j}^{*} E\left(Y_{\alpha(i+1)+j+1}^{s}\right)\right\} d x
$$

Henceforth, $Y_{\gamma}$ denotes the exp-G distribution with power parameter $(\gamma)$.

\subsection{Residual life and reversed residual life functions}

The $n$th moment of the residual life, say $m_{n}(t)=E\left[(X-t)^{n} \mid X>t\right], n=1,2$, uniquely determined $F(x)$. The $n$th moment of the residual life of $X$ is given by $m_{n}(t)=\frac{1}{R(t)} \int_{t}^{\infty}(x-t)^{n} d F(x)$. Therefore,

$$
m_{n}(t)=\frac{1}{R(t)} \sum_{r=0}^{n}\left(\begin{array}{l}
n \\
r
\end{array}\right)(-t)^{n-r} \sum_{i, j=0}^{\infty}\left\{\begin{array}{c}
\Upsilon_{i, j} \int_{t}^{\infty} x^{r} \pi_{\alpha(i+1)+j}(x) d x \\
-Y_{i, j}^{*} \int_{t}^{\infty} x^{r} \pi_{\alpha(i+1)+j+1}(x) d x
\end{array}\right\}
$$

Another interesting function is the mean residual life (MRL) function or the life expectation at age $t$ defined by $m_{1}(t)=E[(X-t) \mid X>t]$, which represents the expected additional life length for a unit which is alive at age $t$. The MRL of $X$ can be obtained by setting $n=1$ in the last equation. The $n$th moment of the reversed residual life, say $M_{n}(t)=E\left[(t-X)^{n} \mid X \leq t\right]$ for $t>0$ and $n=1,2, \ldots$ uniquely determines $F(x)$. We obtain $M_{n}(t)=$ $\frac{1}{F(t)} \int_{0}^{t}(t-x)^{n} d F(x)$. Then, the $n$th moment of the reversed residual life of $X$ becomes

$$
M_{n}(t)=\frac{1}{F(t)} \sum_{r=0}^{n}(-1)^{r}\left(\begin{array}{l}
n \\
r
\end{array}\right) t^{n-r} \sum_{i, j=0}^{\infty}\left\{\begin{array}{c}
Y_{i, j} \int_{0}^{t} x^{r} \pi_{\alpha(i+1)+j}(x) d x \\
-Y_{i, j}^{*} \int_{0}^{t} x^{r} \pi_{\alpha(i+1)+j+1}(x) d x
\end{array}\right\} .
$$

The mean inactivity time (MIT) or mean waiting time (MWT) also called the mean reversed residual life function is given by $M_{1}(t)=E[(t-X) \mid X \leq t]$, and it represents the waiting time elapsed since the failure of an item on condition that this failure had occurred in $(0, t)$. The MIT of the PTL-G family of distributions can be obtained easily by setting $n=1$ in the above equation.

\subsection{Moments, incomplete moments and generating function}

The $r$ th ordinary moment of $X$ is given by $\mu_{r}^{\prime}=E\left(X^{r}\right)=\int_{-\infty}^{\infty} x^{r} f(x) d x$. Then we obtain

$$
\mu_{r}^{\prime}=\sum_{i, j=0}^{\infty}\left\{Y_{i, j} E\left(Y_{\alpha(i+1)+j}^{r}\right)-Y_{i, j}^{*} E\left(Y_{\alpha(i+1)+j+1}^{r}\right)\right\}
$$

Setting $r=1$ in (11), we have the mean of $X$. The last integration can be computed numerically for most parent distributions. The skewness and kurtosis measures can be calculated from the ordinary moments using well-known relationships. The $n$th central moment of $X$, say $M_{n}$, follows as $M_{n}=E(X-\mu)^{n}=\sum_{h=0}^{n}(-1)^{h}\left(\begin{array}{l}n \\ h\end{array}\right)\left(\mu_{1}^{\prime}\right)^{n} \mu_{n-h}^{\prime}$. The main applications of the first incomplete moment refer to the mean deviations and the Bonferroni and Lorenz curves. These curves are very useful in economics, reliability, demography, insurance and medicine. The $s$ th incomplete moment, say $\phi_{s}(t)$, of $X$ can be expressed from (9) as $\phi_{s}(t)=\int_{-\infty}^{t} x^{s} f(x) d x$. Then 


$$
\phi_{s}(t)=\sum_{i, j=0}^{\infty}\left\{\begin{array}{cc}
\Upsilon_{i, j} \int_{-\infty}^{t} & x^{s} \pi_{\alpha(i+1)+j}(x) d x \\
-\Upsilon_{i, j}^{*} \int_{-\infty}^{t} & x^{s} \pi_{\alpha(i+1)+j+1}(x) d x
\end{array}\right\} .
$$

The mean deviations about the mean $\left[\delta_{1}=E\left(\left|X-\mu_{1}^{\prime}\right|\right)\right]$ and about the median $\left[\delta_{2}=E(|X-M|)\right]$ of $X$ are given by $\delta_{1}=2 \mu_{1}^{\prime} F\left(\mu_{1}^{\prime}\right)-2 \phi_{1}\left(\mu_{1}^{\prime}\right)$ and $\delta_{2}=\mu_{1}^{\prime}-2 \phi_{1}(M)$, respectively, where $\mu_{1}^{\prime}=E(X), M=\operatorname{Median}(X)=$ $Q(0.5)$ is the median, $F\left(\mu_{1}^{\prime}\right)$ is easily calculated from (5) and $\phi_{1}(t)$ is the first incomplete moment given by (12) with $s=1$. Ageneral equation for $\phi_{1}(t)$ can be derived from (12) as

$$
\phi_{1}(t)=\sum_{i, j=0}^{\infty}\left\{Y_{i, j} \mathbf{J}_{\alpha(i+1)+j}(x) d x-Y_{i, j}^{*} \mathbf{J}_{\alpha(i+1)+j+1}(x) d x\right\},
$$

where $\mathbf{J}_{\gamma}(x)=\int_{-\infty}^{t} x \pi_{\gamma}(x) d x$ is the first incomplete moment of the exp-G distribution. The moment generating function (mgf) $M_{X}(t)=E\left(e^{t X}\right)$ of $X$ can be derived from equation (9) as

$$
M_{X}(t)=\sum_{i, j=0}^{\infty}\left\{Y_{i, j} M_{\alpha(i+1)+j}(t)-Y_{i, j}^{*} M_{\alpha(i+1)+j+1}(t)\right\}
$$

where $M_{\gamma}(t)$ is the mgf of $Y_{\gamma}$. Hence, $M_{X}(t)$ can be determined from the exp-G generating function.

\subsection{Order statistics}

Order statistics make their appearance in many areas of statistical theory and practice. Let $X_{1}, \ldots, X_{n}$ be a random sample from the PTL-G family of distributions and let $X_{(1)}, \ldots, X_{(n)}$ be the corresponding order statistics. The PDF of $i$ th order statistic, say $X_{i: n}$, can be written as

$$
f_{i: n}(x)=\frac{f(x)}{B(i, n-i+1)} \sum_{j=0}^{n-i}(-1)^{j}\left(\begin{array}{c}
n-i \\
j
\end{array}\right) F^{j+i-1}(x)
$$

where $B(\cdot, \cdot)$ is the beta function. Substituting (5) and (6) in equation (12) and using a power series expansion,we get

where

$$
f(x) F(x)^{j+i-1}=\sum_{w, m=0}^{\infty}\left\{t_{w, m} \pi_{\alpha(w+1)+m}(x)-t_{w, m}^{*} \pi_{\alpha(w+1)+m+1}(x)\right\},
$$

$$
t_{w, m}=\sum_{k=0}^{\infty} \frac{2^{\alpha(w+1)-m} \alpha \lambda^{w+1}(-1)^{w+m+k}(k+1)^{w}\left(\begin{array}{c}
j+i-1 \\
k
\end{array}\right)\left(\begin{array}{c}
\alpha(w+1)-1 \\
m
\end{array}\right)}{w !\left(1-e^{-\lambda}\right)^{j+i}[\alpha(w+1)+m]}
$$

and

$$
t_{w, m}^{*}=\sum_{k=0}^{\infty} \frac{2^{\alpha(w+1)-m} \alpha \lambda^{w+1}(-1)^{w+m+k}(k+1)^{w}\left(\begin{array}{c}
j+i-1 \\
k
\end{array}\right)\left(\begin{array}{c}
\alpha(w+1)-1 \\
m
\end{array}\right)}{w !\left(1-e^{-\lambda}\right)^{j+i}[\alpha(w+1)+m+1]} .
$$

The PDF of $X_{i}: n$ can be expressed as

$$
f_{i: n}(x)=\sum_{j=0}^{n-i} \frac{(-1)^{j}\left(\begin{array}{c}
n-i \\
j
\end{array}\right)}{B(i, n-i+1)} \sum_{w, m=0}^{\infty}\left\{\begin{array}{c}
t_{w, m} \pi_{\alpha(w+1)+m}(x) \\
-t_{w, m}^{?} \pi_{\alpha(w+1)+m+1}(x)
\end{array}\right\} .
$$

Then, the density function of the PTL order statistics is a mixture of exp-G densities. Based on the last equation, we note that the properties of $X_{i}: n$ follow from those properties of $Y_{\alpha(w+1)+m}$ and $Y_{\alpha(w+1)+m+1}$. For example, the moments of $X_{i}: n$ can be expressed as

$$
E\left(X_{i: n}^{q}\right)=\sum_{j=0}^{n-i} \frac{(-1)^{j}\left(\begin{array}{c}
n-i \\
j
\end{array}\right)}{B(i, n-i+1)} \sum_{w, m=0}^{\infty}\left\{\begin{array}{c}
t_{w, m} E\left(Y_{\alpha(w+1)+m}^{q}\right) \\
-t_{w, m}^{*} E\left(Y_{\alpha(w+1)+m+1}^{q}\right)
\end{array}\right\} .
$$

The L-moments are analogous to the ordinary moments but can be estimated by linear combinations of order statistics. They exist whenever the mean of the distribution exists, even though some higher moments may not exist, and are 
relatively robust to the effects of outliers. Based upon the moments in equation (14), we can derive explicit expressions for the L-moments of $X$ as infinite weighted linear combinations of the means of suitable PTL order statistics. They are linear functions of expected order statistics defined by

$$
\lambda_{r}=\frac{1}{r} \sum_{d=0}^{r-1}(-1)^{d}\left(\begin{array}{c}
r-1 \\
d
\end{array}\right) E\left(X_{r-d}: r\right), r \geq 1 .
$$

\section{Characterizations}

In this section we present certain characterizations of PTL-G distribution. The first characterization is based on hazard function and the second one is in terms of the ratio of two truncated moments.

\subsection{Characterization based on hazard function}

It is known that the hazard function, $h_{F}$, of a twice differentiable distribution function, $F$, satisfies the first order differential equation

$$
\frac{f^{\prime}(x)}{f(x)}=\frac{h_{F}^{\prime}(x)}{h_{F}(x)}-h_{F}(x)
$$

For many univariate continuous distributions, this is the only characterization available in terms of the hazard function. The following characterizations establish a non-trivial characterization for (5) in terms of the hazard function which is not of the trivial form given in (14).

Proposition 4.1.1. Let $X: \Omega \rightarrow R$ be a continuous random variable. Then $X$ has $p d f$ of (5) if and only if its hazard function $h_{F}(x)$ satisfies the differential equation

$$
h_{F}^{\prime}(x)-\frac{g^{\prime}(x) h_{F}(x)}{g(x)}=\frac{2 g(x)}{(\alpha \lambda)^{-1}} \frac{d}{d x}\left\{\frac{(G(x))^{\alpha-1}[2-G(x)]^{\alpha-1} \bar{G}(x)}{1-\exp \left\{\lambda\left((G(x))^{\alpha}[2-G(x)]^{\alpha}-1\right)\right\}}\right\} \text {. }
$$

Proof. If $X$ has $p d f$ of (5), then clearly (4.1.2) holds. Now, if (15) holds, then

$$
\frac{d}{d x}\left\{(g(x))^{-1} h_{F}(x)\right\}=2 \alpha \lambda \frac{d}{d x}\left\{\frac{(G(x))^{\alpha-1}[2-G(x)]^{\alpha-1} \bar{G}(x)}{1-\exp \left\{\lambda\left((G(x))^{\alpha}[2-G(x)]^{\alpha}-1\right)\right\}}\right\},
$$

or

$$
h_{F}(x)=\frac{2 \alpha \lambda g(x)(G(x))^{\alpha-1}[2-G(x)]^{\alpha-1} \bar{G}(x)}{1-\exp \left\{\lambda\left((G(x))^{\alpha}[2-G(x)]^{\alpha}-1\right)\right\}}, x \in R,
$$

which is the hazard function of (5).

Remark 4.1.1. For $\alpha=1$, the differential equation (15) will have the following form

$$
\begin{aligned}
& h_{F}^{\prime}(x)-\frac{g^{\prime}(x) h_{F}(x)}{g(x)}=\frac{2 \lambda(g(x))^{2}}{(1-\exp \{\lambda((G(x))[1+\bar{G}(x)]-1)\})^{2}} \\
& \times\{(2 G(x) \bar{G}(x)+1) \exp \{\lambda((G(x))[1+\bar{G}(x)]-1)\}-1\} .
\end{aligned}
$$

\subsection{Characterizations in terms of two truncated moments}

In this subsection we present characterizations of (5) in terms of a simple relationship between two truncated moments. Our first characterization result employs a theorem due to Glänzel (1987), see Theorem 1 below. Note that the result holds also when the interval $H$ is not closed. Moreover, it could be also applied when the $c d f F$ does not have a closed form. As shown in Glänzel (1990), this characterization is stable in the sense of weak convergence.

Theorem 1. Let $(\Omega, F, P)$ be a given probability space and let $H=[d, e]$ be an interval for some $d<e$ ( $d=-\infty, e=\infty$ might as well be allowed). Let $X: \Omega \rightarrow H$ be a continuous random variable with the distribution function $F$ and let $q_{1}$ and $q_{2}$ be two real functions defined on $H$ such that 


$$
E\left[q_{2}(X) \mid X \geq x\right]=E\left[q_{1}(X) \mid X \geq x\right] \eta(x), \quad x \in H,
$$

is defined with some real function $\eta$. Assume that $q_{1}, q_{2} \in C^{1}(H), \eta \in C^{2}(H)$ and $F$ is twice continuously differentiable and strictly monotone function on the set $H$. Finally, assume that the equation $\eta q_{1}=q_{2}$ has no real solution in the interior of $H$. Then $F$ is uniquely determined by the functions $q_{1}, q_{2}$ and $\eta$, particularly

$$
F(x)=\int_{a}^{x} C\left|\frac{\eta^{\prime}(u)}{\eta(u) q_{1}(u)-q_{2}(u)}\right| \exp (-s(u)) d u,
$$

where the function $s$ is a solution of the differential equation $s^{\prime}=\frac{\eta^{\prime} q_{1}}{\eta q_{1}-q_{2}}$ and $C$ is the normalization constant, such that $\int_{H} d F=1$.

Here is our characterization.

Proposition 4.2.1. Let $X: \Omega \rightarrow R$ be a continuous random variable and let

and

$$
q_{1}(x)=\exp \left\{\lambda(G(x))^{\alpha}[2-G(x)]^{\alpha}\right\}
$$

$$
q_{2}(x)=q_{1}(x)(G(x)[2-G(x)])^{\alpha} \text { for } x \in R .
$$

The random variable $X$ belongs to the family (5) if and only if the function $\eta$ defined in Theorem1 has the form

$$
\eta(x)=\frac{1}{2}\left\{1+(G(x)[2-G(x)])^{\alpha}\right\}, \quad x \in R .
$$

Proof. Let $X$ be a random variable with $p d f(5)$, then

$$
(1-F(x)) E\left[q_{1}(x) \mid X \geq x\right]=\frac{\lambda}{1-e^{-\lambda}}\left\{1-(G(x)[2-G(x)])^{\alpha}\right\}, \quad x \in R,
$$

and

$$
(1-F(x)) E\left[q_{2}(x) \mid X \geq x\right]=\frac{\lambda}{2\left(1-e^{-\lambda}\right)}\left\{1-(G(x)[2-G(x)])^{2 \alpha}\right\} \quad x \in R,
$$

and finally

$$
\eta(x) q_{1}(x)-q_{2}(x)=\frac{q_{1}(x)}{2}\left\{1-(G(x)[2-G(x)])^{\alpha}\right\}>0 \text { for } x \in R .
$$

Conversely, if $\eta$ is given as above, then

$$
s^{\prime}(x)=\frac{\eta^{\prime}(x) q_{1}(x)}{\eta(x) q_{1}(x)-q_{2}(x)}=\frac{2 \alpha g(x)(G(x)[2-G(x)])^{\alpha-1} \bar{G}(x)}{1-(G(x)[2-G(x)])^{\alpha}}, \quad x \in R,
$$

and hence

$$
s(x)=-\log \left\{(G(x)[2-G(x)])^{\alpha}\right\}, \quad x \in R .
$$

Now, in view of Theorem 1, $X$ has density (5).

Corollary 4.2.1. Let $X: \Omega \rightarrow R$ be a continuous random variable and let $q_{1}(x)$ be as in Proposition (17). Then $X$ has $p d f$ (5) if and only if there exist functions $q_{2}$ and $\eta$ defined in Theorem 1 satisfying the differential equation

$$
\frac{\eta^{\prime}(x) q_{1}(x)}{\eta(x) q_{1}(x)-q_{2}(x)}=\frac{2 \alpha g(x)(G(x)[2-G(x)])^{\alpha-1} \bar{G}(x)}{1-(G(x)[2-G(x)])^{\alpha}}, x \in R .
$$

The general solution of the differential equation in Corollary 4.2.1 is 


$$
\begin{aligned}
& \eta(x)=\left\{1-(G(x)[2-G(x)])^{\alpha}\right\}^{-1} \\
& \times\left[-\int 2 \alpha g(x)(G(x)[2-G(x)])^{\alpha-1}\left(q_{1}(x)\right)^{-1} q_{2}(x) d x+D\right]
\end{aligned}
$$

where $D$ is a constant.

\section{Estimation}

Several approaches for parameter estimation were proposed in the literature but the maximum likelihood method is the most commonly employed. The maximum likelihood estimators (MLEs) enjoy desirable properties and can be used for constructing confidence intervals and regions and also in test statistics. The normal approximation for these estimators in large samples can be easily handled either analytically or numerically. So, we consider the estimation of the unknown parameters of this family from complete samples only by maximum likelihood. Let $x_{1}, \ldots, x_{n}$ be a random sample from the PTL-G distribution with parameters $\delta, a$ and $\psi$. Let $\Theta$ be the $p \times 1$ parameter vector. For determining the MLE of $\Theta$, we have the log-likelihood function

$$
\begin{gathered}
\ell=\ell(\Theta)=n \log (2)+n \log \lambda+n \log \alpha-n \log \left(1-e^{-\lambda}\right) \\
+\sum_{i=1}^{n} \log g\left(x_{i} ; \psi\right)+(\alpha-1) \sum_{i=1}^{n} \log G\left(x_{i} ; \psi\right) \\
+\sum_{i=1}^{n} \quad \log \left[1-G\left(x_{i} ; \psi\right)\right]+(\alpha-1) \sum_{i=1}^{n} \log \left[2-G\left(x_{i} ; \psi\right)\right] \\
-\lambda \sum_{i=1}^{n} G\left(x_{i} ; \psi\right)^{\alpha}\left[2-G\left(x_{i} ; \psi\right)\right]^{\alpha} .
\end{gathered}
$$

The components of the score vector are

$$
\begin{aligned}
& U_{\lambda}=\frac{n}{\lambda}-\frac{n e^{-\lambda}}{\left(1-e^{-\lambda}\right)}-\sum_{i=1}^{n} G\left(x_{i} ; \psi\right)^{\alpha}\left[2-G\left(x_{i} ; \psi\right)\right]^{\alpha}, \\
& U_{\alpha}=\frac{n}{\alpha}+\sum_{i=1}^{n} \log G\left(x_{i} ; \psi\right)+\sum_{i=1}^{n} \log \left[2-G\left(x_{i} ; \psi\right)\right] \\
& -\lambda \sum_{i=1}^{n} \frac{\left\{\log \left[2-G\left(x_{i} ; \psi\right)\right]+\log G\left(x_{i} ; \psi\right)\right\}}{\left[2-G\left(x_{i} ; \psi\right)\right]^{-\alpha} G\left(x_{i} ; \psi\right)^{-\alpha}}
\end{aligned}
$$

and

$$
\begin{gathered}
U_{\psi}=\sum_{i=1}^{n} \frac{g^{\prime}\left(x_{i} ; \psi\right)}{g\left(x_{i} ; \psi\right)}+(\alpha-1) \sum_{i=1}^{n} \frac{G^{\prime}\left(x_{i} ; \psi\right)}{G\left(x_{i} ; \psi\right)} \\
-\sum_{i=1}^{n} \frac{G^{\prime}\left(x_{i} ; \psi\right)}{1-G\left(x_{i} ; \psi\right)}-(\alpha-1) \sum_{i=1}^{n} \frac{G^{\prime}\left(x_{i} ; \psi\right)}{2-G\left(x_{i} ; \psi\right)} \\
-\lambda \alpha \sum_{i=1}^{n} G^{\prime}\left(x_{i} ; \psi\right)\left\{\begin{array}{c}
{\left[2-G\left(x_{i} ; \psi\right)\right]^{\alpha} G\left(x_{i} ; \psi\right)^{\alpha-1}} \\
-G\left(x_{i} ; \psi\right)^{\alpha}\left[2-G\left(x_{i} ; \psi\right)\right]^{\alpha-1}
\end{array}\right\},
\end{gathered}
$$

where $g^{\prime}\left(x_{i} ; \psi\right)=\partial g\left(x_{i} ; \psi\right) / \partial \psi, G^{\prime}\left(x_{i} ; \psi\right)=\partial G\left(x_{i} ; \psi\right) / \partial \psi$. Setting the nonlinear system of equations $U_{\lambda}=U_{a}=$ 0 and $U_{\psi}=0$ and solving them simultaneously yields the MLE. To solve these equations, it is usually more convenient to use nonlinear optimization methods such as the quasi-Newton algorithm to numerically maximize $\ell$.

\section{Simulation of PTL-E distribution}

In this section, we study the performance of the PTL-E distribution by conducting various simulations for different sizes $(\mathrm{n}=50,150,300)$ by using $\mathrm{R}$-Language. We simulate 500 samples for the true parameters values I: $\lambda=4, \alpha=$ $5.5, \gamma=0.5$ in order to obtain average estimates (AEs), biases and mean square errors (MSEs) of the parameters. They are listed in Table 1. The small values of the biases and MSEs indicate that the maximum likelihood method performs quite well in estimating the model parameters of the proposed distribution. 


\section{Application}

In this section, we use a real data set to show that the PTL-Weibull distribution can be a better model than one based on Beta-exponential, Kumaraswamy-exponential, PTL-Weibull, Modified Weibull and Weibull distribution. The data set given below and represents the failure times of 50 components (per 1000h)

$\begin{array}{cccccccccc}0.036 & 0.058 & 0.061 & 0.074 & 0.078 & 0.086 & 0.102 & 0.103 & 0.114 & 0.116 \\ 0.148 & 0.183 & 0.192 & 0.254 & 0.262 & 0.379 & 0.381 & 0.538 & 0.570 & 0.574 \\ 0.590 & 0.618 & 0.645 & 0.961 & 1.228 & 1.600 & 2.006 & 2.054 & 2.804 & 3.058 \\ 3.076 & 3.147 & 3.625 & 3.704 & 3.931 & 4.073 & 4.393 & 4.534 & 4.893 & 6.274 \\ 6.816 & 7.896 & 7.904 & 8.022 & 9.337 & 10.940 & 11.020 & 13.880 & 14.730 & 15.080\end{array}$

The values in table Tab1 indicate that the PTL-W distribution leads to a better fit than the Weibull distribution, modified Weibull distribution, exponential distribution, beta exponential distribution, Kumaraswamy exponential distribution and PTL-E distribution. Also, from Table 2 PTL-G family of distribution is better than Beta-G and Kumaraswamy-G distribution.

Many other useful real data sets can be found and analyzed see Ibrahim (2019), Ibrahim et al. (2019), Ibrahim (2020a, b), Mansour et al. (2020) and Ibrahim and Yousof (2020).

\section{Concluding remarks}

We have proposed and presented results on a new family of distributions called Poisson Topp Leone-G family of distributions. This family of distributions have applications in Reliability, Economics and Survival data analysis. Properties of this family are studied including reliability properties, quantile function, series expansion of CDF and PDF, moments, moment generating function, mean deviation. Expression for $i^{\text {th }}$ order statistics is given, and estimation of parameters are carried out by Maximum likelihood method. A special sub-model is discussed in detail for illustration propose. Finally, an application is carried out on real data set to check the performance of the proposed family which provides consistently better fit than other models.

\section{References}

1. Aryal, G. R. and Yousof, H. M. (2017). The exponentiated generalized-G Poisson family of distributions. Economic Quality Control, 32(1), 1-17.

2. Brito, E., Cordeiro, G. M., Yousof, H. M., Alizadeh, M. and Silva, G. O. (2017). Topp-Leone Odd LogLogistic Family of Distributions, Journal of Statistical Computation and Simulation, 87(15), 3040-3058.

3. Cordeiro, G. M., Yousof, H. M., Ramires, T. G. and Ortega, E. M. M. (2018). The Burr XII system of densities: properties, regression model and applications. Journal of Statistical Computation and Simulation, 88(3), 432-456.

4. Eugene, N., Lee, C. and Famoye, F. (2002). Beta-normal distribution and its applications. Commun. Stat. Theory Methods, 31, 497-512.

5. Glänzel, W. (1987). A characterization theorem based on truncated moments and its application to some distribution families, Mathematical Statistics and Probability Theory (Bad Tatzmannsdorf, 1986), Vol. B, Reidel, Dordrecht, 75-84.

6. Glänzel, W. (1990). Some consequences of a characterization theorem based on truncated moments, Statistics: A Journal of Theoretical and Applied Statistics, 21, 613-618.

7. Gupta, R. C., Gupta, P. L. and Gupta, R. D. (1998). Modeling failure time data by Lehmann alternatives. Commun. Stat. Theory Methods, 27, 887-904.

8. Hamedani, G. G., Altun, E, Korkmaz, M. C., Yousof, H. M. and Butt, N. S. (2018). A new extended G family of continuous distributions with mathematical properties, characterizations and regression modeling. Pak. J. Stat. Oper. Res., 14(3), 737-758.

9. Hamedani, G. G. Rasekhi, M., Najib, S. M., Yousof, H. M. and Alizadeh, M., (2019). Type II general exponential class of distributions. Pak. J. Stat. Oper. Res., XV (2), 503-523.

10. Hamedani, G. G. Yousof, H. M., Rasekhi, M., Alizadeh, M., Najibi, S. M. (2017). Type I general exponential class of distributions. Pak. J. Stat. Oper. Res., XIV (1), 39-55.

11. Ibrahim, M. (2019). A new extended Fréchet distribution: properties and estimation. Pak. J. Stat. Oper. Res.,15 (3), 773-796. 
12. Ibrahim, M. (2020a). The compound Poisson Rayleigh Burr XII distribution: properties and applications. Journal of Applied Probability and Statistics, 15(1), 73-97.

13. Ibrahim, M. (2020b). The generalized odd Log-logistic Nadarajah Haghighi distribution: statistical properties and different methods of estimation. Journal of Applied Probability and Statistics, forthcoming.

14. Ibrahim, M. and Yousof, H. M. (2020). A new generalized Lomax model: statistical properties and applications, Journal of Data Science, 18(1), $190-217$.

15. Ibrahim, M., Yadav, A. S. Yousof, H. M., Goual, H. and Hamedani, G. G. (2019). A new extension of Lindley distribution: modified validation test, characterizations and different methods of estimation, Communications for Statistical Applications and Methods, 26(5), 473-495.

16. Marshall, A. W. and Olkin, I. (1997). A new method for adding a parameter to a family of distributions with application to the Exponential and Weibull families. Biometrika, 84, 641-652.

17. Korkmaz, M. C. Yousof, H. M. and Hamedani G. G. (2018a). The exponential Lindley odd log-logistic $\mathrm{G}$ family: properties, characterizations and applications. Journal of Statistical Theory and Applications, 17(3), $554-571$.

18. Korkmaz, M. C., Yousof, H. M., Hamedani G. G. and Ali, M. M. (2018b). The Marshall-Olkin generalized G Poisson family of distributions, Pakistan Journal of Statistics, 34(3), 251-267.

19. Mansour, M., Yousof, H. M., Shehata, W. A. M. and Ibrahim, M. (2020). A new two parameter Burr XII distribution: properties, copula, different estimation methods and modeling acute bone cancer data, Journal of Nonlinear Science and Applications, 13, 223-238.

20. Merovci, F., Alizadeh, M., Yousof, H. M. and Hamedani G. G. (2017). The exponentiated transmuted$\mathrm{G}$ family of distributions: theory and applications, Communications in Statistics-Theory and Methods, 46(21), 10800-10822.

21. Nascimento, A. D. C., Silva, K. F., Cordeiro, G. M., Alizadeh, M. and Yousof, H. M. (2019). The odd Nadarajah-Haghighi family of distributions: properties and applications. Studia Scientiarum Mathematicarum Hungarica, 56(2), 1-26.

22. Nofal, Z. M., Afify, A. Z., Yousof, H. M. and Cordeiro, G. M. (2017). The generalized transmuted-G family of distributions. Communications in Statistics-Theory and Method, 46, 4119-4136.

23. Rezaei, S., B. B. Sadr, M. Alizadeh, and S. Nadarajah. 2017. Topp-Leone generated family of distributions: Properties and applications. Communications in Statistics: Theory and Methods 46 (6), 2893-2909.

24. Weibull, W. (1951). A statistical distribution function of wide applicability. J. Appl. Mech. Trans, 18, 293--297.

25. Yousof, H. M., Afify, A. Z., Alizadeh, M., Butt, N. S., Hamedani, G. G. and Ali, M. M. (2015). The transmuted exponentiated generalized-G family of distributions, Pak. J. Stat. Oper. Res., 11, 441-464.

26. Yousof, H. M., Afify, A. Z., Hamedani, G. G. and Aryal, G. (2017). The Burr X generator of distributions for lifetime data. Journal of Statistical Theory and Applications, 16, 288-305.

27. Yousof, H. M., Altun, E., Ramires, T. G., Alizadeh, M. and Rasekhi, M. (2018). A new family of distributions with properties, regression models and applications, Journal of Statistics and Management Systems, 21(1), 163-188.

28. Yousof, H. M., Mansoor, M. Alizadeh, M., Afify, A. Z., Ghosh, I. and Afify, A. Z. (2020). The Weibull-G Poisson family for analyzing lifetime data. Pak. J. Stat. Oper. Res., 16 (1), 131-148. 


\section{Appendix}

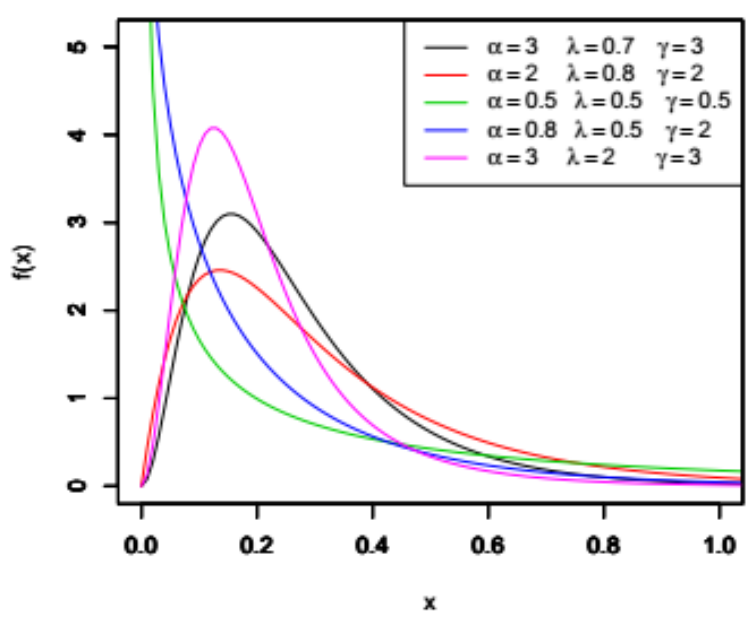

Figure 1: The PDF's of various PTL-E distributions.

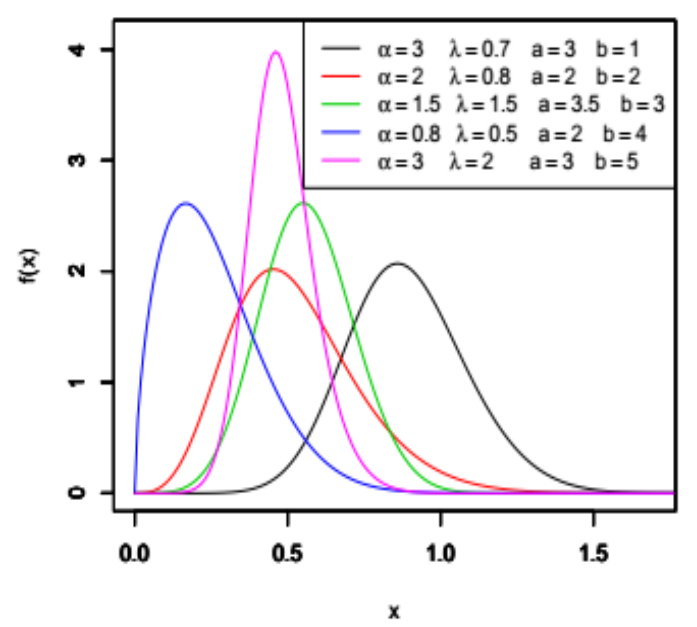

Figure 3: The PDF's of various PTL-W distributions.

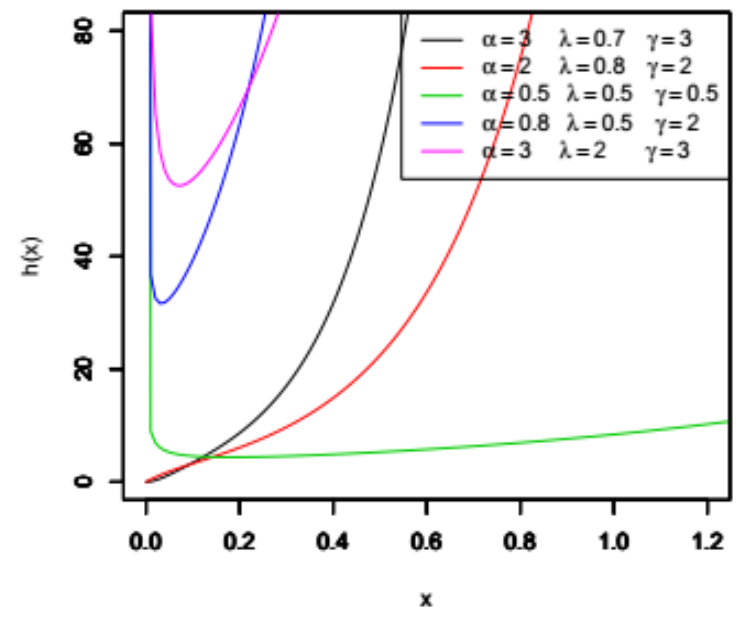

Figure 2: The hazard functions of various PTL-E distributions.

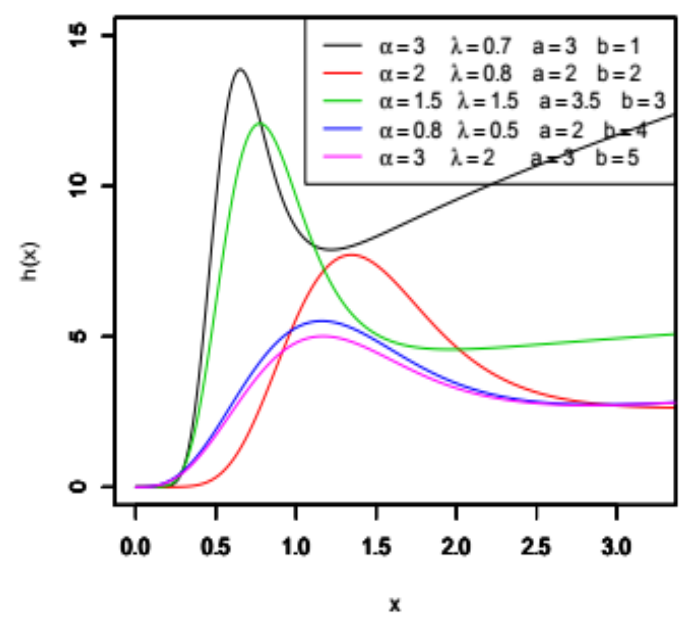

Figure 4: The hazard functions of various PTL-W distributions.

Table 1: Estimated AEs, Biases, and MSEs of the MLEs of parameters of

PTL-E distribution based on 500

simulations of with $n=50,150$ and 300 .

\begin{tabular}{ccccc}
\hline $\mathbf{5 0}$ & parameters & A.E & Bias & MSE \\
\hline \multirow{4}{*}{$\mathbf{1 5 0}$} & $\alpha$ & 3.351 & 0.759 & 1.763 \\
& $\lambda$ & 5.010 & 0.510 & 0.351 \\
& $\gamma$ & 0.735 & 0.335 & 0.073 \\
& $\alpha$ & 3.559 & 0.551 & 0.651 \\
$\mathbf{3 0 0}$ & $\lambda$ & 5.953 & 0.553 & 0.355 \\
& $\gamma$ & 0.776 & 0.376 & 0.103 \\
& $\alpha$ & 3.368 & 0.633 & 0.539 \\
& $\lambda$ & 5.996 & 0.596 & 0.310 \\
& $\gamma$ & 0.755 & 0.355 & 0.083 \\
\hline
\end{tabular}


Table 2: Estimated parameters for the failure times of 50 components(per 1000h).

\begin{tabular}{|c|c|c|c|c|c|}
\hline Model & Estimates & Standard Error & $-\log \mathrm{L}$ & AIC & $\mathrm{K}-\mathrm{S}$ \\
\hline Weibull & $\begin{array}{l}\alpha=0.429 \\
\lambda=0.638\end{array}$ & $\begin{array}{l}0.104 \\
0.073\end{array}$ & 103.0572 & 210.114 & 0.175 \\
\hline Modified Weibull & $\begin{array}{l}\alpha=0.043 \\
\beta=0.492 \\
\gamma=0.619\end{array}$ & $\begin{array}{l}0.131 \\
0.181 \\
0.154\end{array}$ & 102.320 & 210.640 & 0.138 \\
\hline PTL-Weibull & $\begin{array}{c}\lambda=2.118 \\
\alpha=0.146 \\
a=3.902 \\
b=1.3 \times 10^{-5}\end{array}$ & $\begin{array}{c}0.899 \\
0.024 \\
0.021 \\
0.13 \times 10^{-5}\end{array}$ & 100.818 & 209.636 & 0.122 \\
\hline Exponential & $\lambda=0.299$ & 0.042 & 110.342 & 222.685 & 0.478 \\
\hline Beta-Exponential & $\begin{array}{l}a=0.529 \\
b=0.717 \\
\lambda=0.281\end{array}$ & $\begin{array}{l}0.101 \\
0.308 \\
0.132\end{array}$ & 102.358 & 210.716 & 0.144 \\
\hline $\begin{array}{c}\text { Kumaraswamy- } \\
\text { Exponential }\end{array}$ & $\begin{array}{l}a=0.605 \\
b=3.274 \\
\lambda=0.044\end{array}$ & $\begin{array}{l}0.204 \\
1.308 \\
0.012\end{array}$ & 102.166 & 210.332 & 0.131 \\
\hline PTL-Exponential & $\begin{array}{l}\lambda=0.823 \\
\alpha=0.606 \\
\gamma=0.083\end{array}$ & $\begin{array}{l}0.304 \\
0.122 \\
0.029\end{array}$ & 102.094 & 210.189 & 0.128 \\
\hline
\end{tabular}

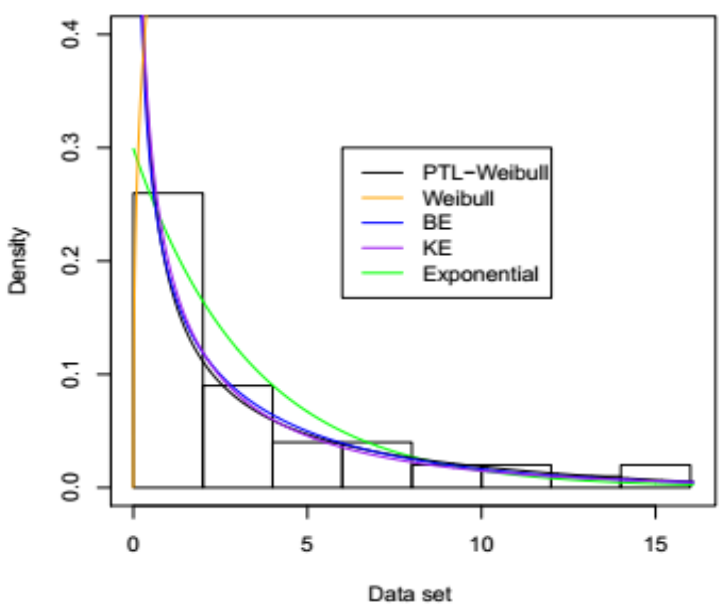

Figure 5: Estimated densities of the models for data set.

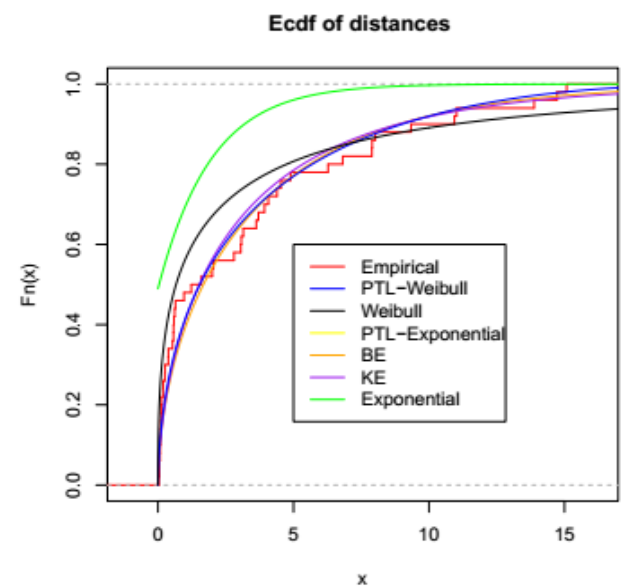

Figure 6: Fitted CDFs plots of the considered distribution for the real data set. 

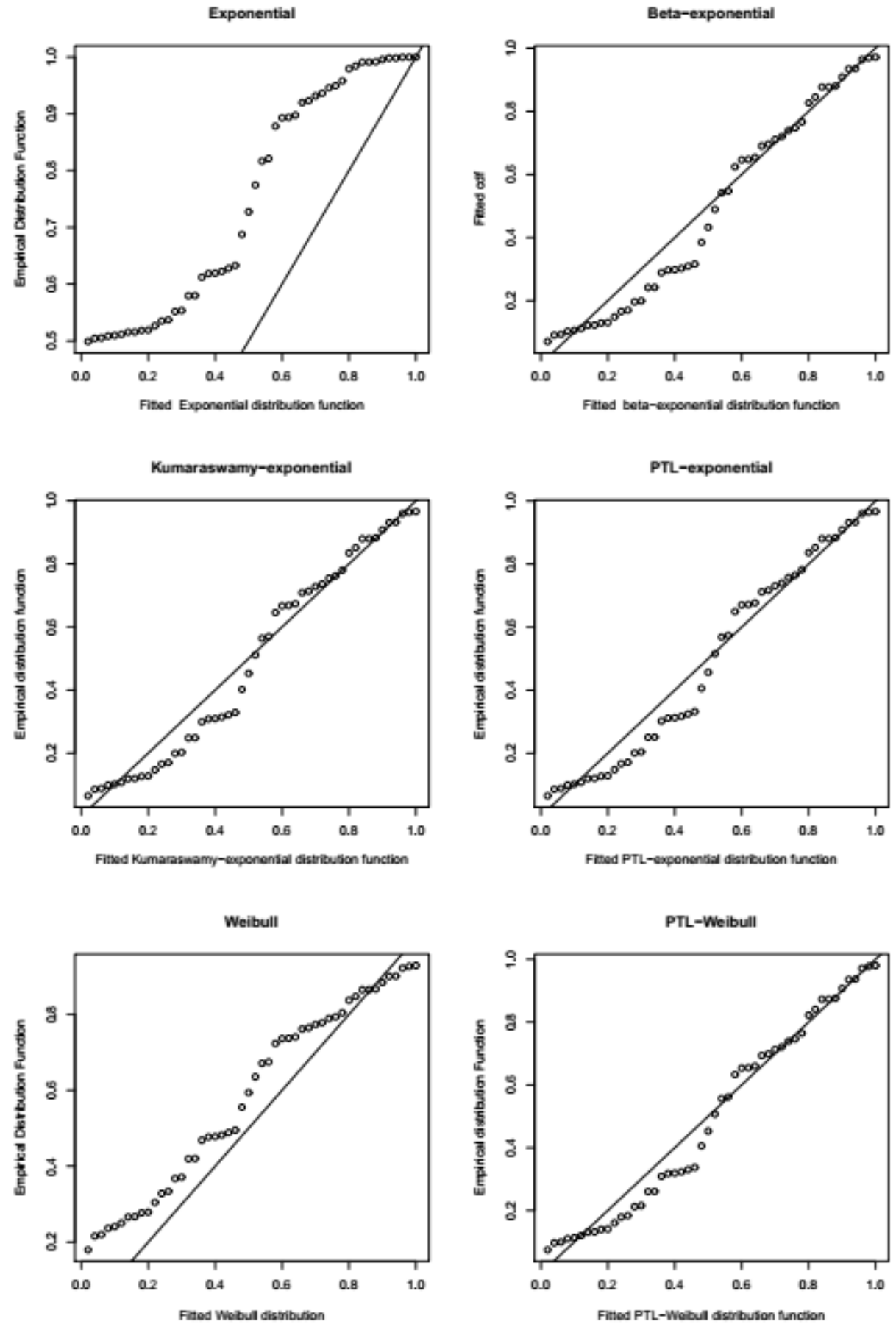

Figure 7: The P-P plots for the real data set 